\title{
Increasing the protein and gluten content in the grain of bread wheat using marker-assisted selection
}

\author{
Morozova E.V.* , Pshenichnikova T.A., Simonov A.V., Shchukina L.V. \\ Institute of Cytology and Genetics, SB RAS, Novosibirsk, Russia \\ *e-mail:emorozova@bionet.nsc.ru
}

Yeast bread-baking is possible due to the gluten, which creates a three-dimensional frame that holds gas bubbles inside the raw dough. The grade of grain and its use for technological purposes are determined according to the amount of gluten in the grain and parameters of dough elasticity and extensibility. Since the amount of gluten closely correlates with the amount of protein in the grain, the percentage of gluten determines the nutritional value of bakery products. In our work, we studied the collection of cultivars of domestic selection for the raw gluten content in grain (RGCG). A correlation analysis was carried out between the alleles of 24 microsatellite $(\mathrm{Xgwm})$ markers located on different chromosomes and RGCG percentage. One of the highest values of the RGCG was associated with the Xgwm261 marker allele with a length of 185 base pairs. The marker is located on chromosome 2 of the D-genome of wheat, in the short arm. To verify the role of this chromosome region in the formation of a high RGCG, a chromosome with the above-mentioned allele was introduced into the well-studied cultivar Saratovskaya 29 (S29) by backcrossing to the monosomic line for $2 \mathrm{D}$ chromosome. The donors of the allele associated with a high content of gluten were the old cultivars Sibirka 1818 and Cesium 111 bred in Siberia. By this way the single chromosome inter-varietal substitution lines S29 (Sibirka 1818 2D) and S29 (Cesium 111 2D) were obtained. The interim control of the gluten content during the backcrosses showed that the lines exceed the recipient cultivar for this trait.

Acknowledgements: This study was funded by the budjet ICG project No. 0324-20190039 . 